Reading only this book, one would conclude that all is well in the nuclear industry. It does discuss the major public concerns about reactor safety, terrorism, and disposal of long-lived radioactive wastes, but being written from an establishment point of view, it gives the impression that everything is under control, or at least that research is in progress which will satisfactorily solve these problems.

The public does have an often irrational fear of radioactivity, but there are legitimate questions of safety and publio policy raised by the environmentalists which have not satisfactorily been answered by the nuclear establishment. For a review of these questions, I would recommend Ian Breach's excellent book on the Windscale Inquiry (Windscale Fallout. A Primer for the Age of Nuclear Controversy, Penguin, 1978, £0.90p).

\section{Mutagen-induced Chromosome Damage in Man}

Edited by H. J. Evans \& D. C. Lloyd. Pp. 355, illustrated. Edinburgh: University Press, 1978. $£ 15$.

This book gives the texts of 37 invited papers presented at an international symposium held in Edinburgh in July 1977. They reflect strongly the important advances in cytogenetic technique (in particular the chromosomal banding methods, and the differential staining obtainable following bromodeoxyuridine incorporation) which have occurred in the 11 intervening years since the previous Edinburgh conference. Within the boundaries indicated by the title, the coverage of topics is very wide, and gives evidence of the lively interest in this field of research.

As is to be expected, the stimulated blood lymphocyte system continues to play a dominant role in the direct assignment of damage to human chromosomes both for ionizing radiation (8 papers) and for chemical or physical mutagens (6 papers). Many of these papers touch on the problems of obtaining reliable quantitative information, and of interpreting data, stressing the fact that the lymphocyte cell system is not quite as simple as was assumed in the early days of its use. This is particularly pertinent when intercomparison between species or between different inducing agents is made ( 3 papers).

Following radio- or chemotherapy, many normal tissues sustain chromosomal changes and these may survive for many years after the cessation of treatment. Assessment of the degree of damage and possible somatic consequences form the subject for 6 papers, and another considers the question of non-disjunction, a consequence which has a bearing on the next generation.

Cells from individuals with abnormal karyotypes, as well as some forms of cancer cells are now known to have an zlevated radiosensitivity with respect to aberration induction and 5 papers are devoted to this topic.

The improved techniques for observing and quantifying the particular type of aberration known as sister-chromatid exchange have made this an important tool for mutagen screening. Five papers are specifically concerned with the application of the method for detection of damage, but the method is also used in a subsidiary manner in several investigations reported in the other papers.

Aberration scoring involves considerable time and effort (not to mention expertise) and there have been numerous attempts to devise some method of automation, particularly in cases of population screening. The 3 concluding papers discuss and evaluate the current position of computer analysis including the promising new field of flow cytometry.

The relatively short time which has elasped between the symposium and this publication means that a reasonably comprehensive picture can be obtained of current thinking in this field. Certainly the usefulness and potential of chromosomal analysis for assessing the hazards of mutagens and for probing the biology of cellular genetic mechanisms is ably illustrated in this volume.
Operative Surgery. Fundamental International Techniques. Paediatric Surgery (3rd edn)

Edited by H. Homewood Nixon. General Editors: C. Rob and Sir Rodney Smith. Associate Editor: H. Dudley. Pp. 489, Illustrated. London: Butterworths, 1978. $£ 42.50$.

The editor of this comprehensive tome on operative paediatric surgery can be congratulated. Correlation and co-ordination of the material from 46 contributors must have been a formidable task.

The contributions come predominantly from paediatric surgeons. There are a number of chapters written by surgeons specializing in a certain section of the field, but there is little that would not be considered by a trained paediatric surgeon to be within his competence. There are a number of chapters, notably those on liver resection and adrenalectomy written by surgeons whose practice is predominantly adult. These bring valuable expertise, but it is a great pity they could not have been considered in the context of childhood disease. Others, bladder, neck resection and meatoplasty, have little relevance to paediatric surgery.

Sections on hiatus hernia, duodenal and small bowel atresia, meconium ileus, duplication of the alimentary tract and inflammatory bowel diseases are particularly valuable. Oesophageal atresia and oesophageal replacements are well covered as are sacrococcygeal teratoma, thyroglossal and branchial cysts. Hodgkin's disease receives more general attention. Splenectomy in staging, with its risks of subsequent infection, must be debatable. Nephrectomy is well covered as is the segment on renal tumours, although the reviewer failed to find any comment on the management of the not uncommon bilateral nephroblastoma.

The large subjects of imperforate anus, biliary atresia, treatment of neuroblastoma, receive a more general survey. The various procedures for treating cleft palate, bat ears and hypospadias are extensively reviewed. More details of operative techniques, here, could have been helpful. More information on the different types of diaphragmatic hernia, malrotation (this good account is confined to the standard type) and spinal dysraphism would have been valuable.

Nothing is said about the methods of treating the higher rectal and colonic polyps, with diathermy snare through the sigmoidoscope. The content of the chapter on the treatment of hydrocephalus suggests this was written some time before the book went to the press. Perhaps the author should have been allowed to up-date his contribution.

Most of the remaining contributions describe satisfactory, standard, procedures. The contributions which stand out for ingenuity are the treatment of co-anal atresia (with a Burrended drill) and the approach to an inguinal hernia, through the external oblique, without interfering with the external ring.

Although there is room for a difference of opinion, two contributions leave the reviewer with a sense of disquiet: the first is the inclusion of para-umbilical hernia with epigastric herniae and the recommendation that the former should be treated "as there is no natural history of spontaneous cure". Many paediatric surgeons have observed the "spontaneous cure" in the majority of para-, as well as true umbilical hernias; most of these well before the age of 5 years.

The second is the procedure to divide the sternocleidomastoid through the middle third for sternomastoid torticollis. The accessory nerve often runs through the muscle and provides the main motor supply to its fibres. The procedure described does not appear to offer any great advantage.

The illustrations are of varying quality and some are difficult to interpret. Surgeons who have learnt their anatomy in the last $\mathbf{1 5}$ years will need to familiarize themselves with some of the older terminology.

This book will be valuable to the paediatric surgeon in training and to the adult surgeon with an interest in 